\title{
miR-22-Notch Signaling Pathway Is Involved in the Regulation of the Apoptosis and Autophagy in Human Ovarian Cancer Cells
}

\author{
Yan $\mathrm{Li}^{a}{ }^{a}$ Yanjun $\mathrm{Gu},{ }^{*}, b$ Na Tang, ${ }^{a}$ Yanqing Liu, ${ }^{b}$ and Zhankao Zhao ${ }^{b}$ \\ ${ }^{a}$ Department of Obstetrics and Gynecology, Tianjin Union Medicine Center; Tianjin 300121, China: and ${ }^{b}$ Department \\ of Pathology, Affiliated Hospital of Logistics University of People's Armed Police Force (PAPF); Tianjin 300162, \\ China. \\ Received February 2, 2018; accepted May 15, 2018
}

microRNA-22 (miR-22) is a brain-enriched regulatory gene which has been reported to be involved in the development of cancers. The Notch signaling pathway exerts important functions in cell growth. This study is designed to investigate the mechanisms of miR-22-Notch signaling pathway in apoptosis and autophagy of human ovarian cancer cells. After over-expressing miR-22 in human ovarian cancer cell lines OVCAR-3 and SKOV3, cell viability is determined by 2,3-bis-(2-methoxy-4-nitro-5-sulfophenyl)-2H-tetrazolium-5-carboxanilide (XTT) method, cell apoptosis is observed by Flow cytometry (FCM), mRNA expression of miR-22 is measured by RNA preparation and RT-PCR, protein expression of Notch1, Hes1, Beclin1 and LC3B-II is analyzed by Western blot. It is suggested that miR-22 expression is heavily decreased in human ovarian cancer cell lines OVCAR-3 and SKOV3. Over-expression of miR-22 potently suppresses cell viability and authophagy while promotes the percentage of apoptotic cancer cells. In addition, the decreased expression level of Notch1 and its targeted gene is detected in miR-22-over-expressed cells. Moreover, followed by the block of the Notch signaling pathway using Notch1 small interference RNA (siRNA), the effects of miR-22 on the apoptosis and autophagy of human ovarian cancer cell lines OVCAR-3 and SKOV3 are obviously blocked. Together, miR-22 inhibits apoptosis and promotes autophagy of human ovarian cancer cells through the suppression of the Notch signaling pathway, indicating a potential use of miR-22 in the ovarian cancer treatment.

Key words microRNA-22 (miR-22); human ovarian cancer cell; apoptosis; autophagy; Notch

Ovarian cancer is the second main cause of death among gynecologic cancers in women. ${ }^{1)}$ Using surgery and chemotherapy methods, the survival rates of women diagnosed with ovarian cancer have been enhanced. ${ }^{2)}$ However, the prognosis of patients at late-stage is still poor because of the lack of efficient screening and early diagnosis. ${ }^{3)}$ So, it is crucial to elucidate cellular and molecular characteristics of ovarian cancer in order to develop new treatment drugs.

microRNAs (miRNAs) are small non-coding RNA molecules, which are around 22-25 nucleotides in length. miRNAs have received considerable attention because of their multiple cellular functions such as the regulation of cell proliferation, apoptosis and autophagy. The deregulation of miRNAs has become a hallmark of human diseases including cancers. ${ }^{4,5)}$ miR-22, a kind of miRNAs, is a novel regulator of tumors and is regarded as a good diagnostic biomarker for early detection of cancers such as gastric cancer, ${ }^{6)}$ pancreatic cancer, ${ }^{7)}$ rhabdomyosarcoma, ${ }^{8)}$ breast cancer, ${ }^{9)}$ prostate cancer $^{10)}$ and osteosarcoma. ${ }^{11)}$ Furthermore, miR-22 contributes to cellular differentiation, migration and invasion by regulating its downstream transcription factors. ${ }^{12,13)}$

Notch pathway is an evolutionarily conserved signaling pathway which is activated to release the intracellular domain of Notch (NICD) to activate the Notch target genes Hes-1 and c-Myc. The Notch family consists of four receptors and five ligands. ${ }^{14)}$ The Notch signaling pathway is a fundamental signaling mechanism operating in most organisms and cells in the body. The appropriate level of Notch signaling is important for differentiation and tissue homeostasis. $\left.{ }^{15}\right)$ Most importantly, Notch pathway has been proposed to affect proliferation, differentiation and apoptosis in different kinds of cancer cells. ${ }^{16)}$ As reported in current research, pharmacologic inhibition of the Notch pathway is regarded as a potentially experimental approach for the treatment of cancers including ovarian cancer. ${ }^{17)}$

There was ever a report providing the evidence that miR-22 expression is heavily decreased in epithelial ovarian cancer. ${ }^{18)}$ To further demonstrate the functions of miR-22 in ovarian cancer, using cultured ovarian cancer cell lines OVCAR-3 and SKOV3, we discover that miR-22 upregulation is responsible for the reduction of autophagy and the promotion of apoptosis in ovarian cancer cells by targeting the Notch signal pathway. Therefore, miR-22 may be considered as a potential therapeutic target for the treatment of ovarian cancer.

\section{MATERIALS AND METHODS}

Cell Culture The human ovarian cancer cell lines OVCAR-3 and SKOV3, and human normal ovarian cells (ATCC, Manassas, Virginia, U.S.A.) were cultured in RPMI 1640 medium containing $10 \%(\mathrm{v} / \mathrm{v})$ fetal bovine serum (FBS), $10 \mathrm{mmol} / \mathrm{L}$ hydroxyethyl piperazine ethanesulfonic acid, $2 \mathrm{mmol} / \mathrm{L}$ L-glutamine, $50 \mu \mathrm{mol} / \mathrm{L} \beta$-mercaptoethanol, $1 \mu \mathrm{mol} / \mathrm{L}$ sodium pyruvate, $10 \mu \mathrm{g} / \mathrm{mL}$ streptomycin, and $100 \mathrm{U} / \mathrm{mL}$ penicillin (Gibco, New York, NY, U.S.A.) at $37^{\circ} \mathrm{C}$ in a humidified atmosphere of $5 \% \mathrm{CO}_{2}$ in air.

Small Interference RNA (siRNA) Notch1-specific siRNA (Santa Cruz Biotechnology, Santa Cruz, CA, U.S.A.) was shown as follows: GATCCA GGA AGA GTGTTCCTGATT TTCAAGA (sense), GAA ATC AGGAAC ACT CTT CCT TTT TTT GGAAA (anti-sense). For transient transfections, human ovarian cancer cell lines OVCAR-3 and SKOV3 in the expo- 
nential growth phase were grown to $65 \%$ confluence and then transfected with $6 \mu \mathrm{g}$ of Notchl-specific siRNA construct or non-targeting siRNA using HiPerFect (Qiagen, Valencia, CA, U.S.A.). After cells were cultured in medium for $48 \mathrm{~h}$, Western blot analysis was used to evaluate the efficiency of Notch1 siRNA. Furthermore, after cells in the exponential growth phase were grown to $65 \%$ confluence and transfected with Notch siRNA for $12 \mathrm{~h}$, then miR-22 mimics (GenePharma, Shanghai, China) was added in the medium for $48 \mathrm{~h}$, and cell viability, apoptosis and autophagy were investigated.

Cell Proliferation Assay The endogenous effects of miR-22 on cell viability were evaluated using XTT Cell Viability Assay Kit (Sigma, U.S.A.) according to previous study. $^{19)}$

Flow Cytometry (FCM) Cells seeded onto a 96-well plate were treated with miR-22 mimics, Notch1 siRNA or miR-22 mimics plus Notch1 siRNA for $48 \mathrm{~h}$, and the cell apoptotic rate was measured by Annexin V-PE Apoptosis Detection Kits (Beyotime, Shanghai, China) according to previous study. ${ }^{19)}$

Western Blot Analysis Western blot was used to analyze protein expression of Beclin1, LC3B-II, Notch1 and Hes1 after human ovarian cancer cell lines OVCAR-3 and SKOV3 were treated with miR-22 mimics for $48 \mathrm{~h}$ according to the previous study. ${ }^{19)}$ Polyclonal anti-Notch1 (Cell Signaling Technology, MA, U.S.A.) at a dilution of $1: 1000$, Beclin1 (Cell Signaling Technology) at a dilution of 1:2500, LC3B-II (Cell Signaling Technology) at a dilution of $1: 1000$, Notch1 (Cell Signaling Technology) at a dilution of $1: 1500$ and Hes1 (Cell Signaling Technology) at a dilution of 1:2500 were used in this study. Horseradish peroxidase (HRP)-conjugated anti-rabbit immunoglobulin $\mathrm{G}(\mathrm{IgG})$ antibody at a dilution of $1: 1000$ was purchased from Santa Cruz Biotechnology, CA, U.S.A. $\beta$-Actin (dilution of $1: 3000$, Cell Signaling Technology) was used to ensure adequate sample loading for all Western blots. The band density ratio was analyzed with Image J software (National Institutes of Health, Bethesda, Maryland, U.S.A.).

Real Time RT-PCR After human ovarian cancer cell lines OVCAR-3 and SKOV3 were treated with miR-22 mimics for $24 \mathrm{~h}$ and $48 \mathrm{~h}$, total RNA was extracted from cells using TRIzol (Invitrogen, Carlsbad, CA, U.S.A.), reverse transcription was performed using miRNA reverse transcription kits (Applied Biosystems, Foster City, CA, U.S.A.) and the expressions of miR-22 in human ovarian cancer cells were measured by RT-PCR in the ABI 7500 Real-Time PCR system. U6 was used as an internal control for miR-22. Specific primer sequences were synthesized in BIOSUNE Biological Technology Corp (Shanghai, China), and shown as follows: U6: 5'-CGC TTC GGC AGC ACA TAT ACT AAA ATT GGA AC-3' (Forward); 5'-GCT TCA CGA ATT TGC GTG TCA TCC TTGC-3' (Reverse) and miR-22: 5'-ACACTCCAGCTGGGT TCGACGGTC AAC TTC-3' (Forward); 5'-CTC AAC TGG TG T CGT GGA GTC GGC AAT TCA GTT GAG ACA GTT CT-3' (Reverse).

Statistical Analysis Statistical analysis was calculated with one-way ANOVA in SPSS19.0 software (SPSS, Chicago, IL, U.S.A.). Data were expressed as means \pm standard deviation (S.D.) from three independent experiments. $p<0.05$ was considered as a significant difference between the treatment group and the control group.

\section{RESULTS}

Decreased miR-22 Expression Was Observed in Human OVCAR-3 and SKOV3 Cells In cultured human ovarian cancer cell lines OVCAR-3 and SKOV3 and normal ovarian cells, the expression levels of miR-22 were evaluated using real-time RT-PCR. As demonstrated in Fig. 1, it was found that, compared to normal human ovarian cells, miR-22 expression was decreased obviously in both OVCAR-3 and SKOV3 cells after $24 \mathrm{~h}$ and $48 \mathrm{~h}$ of culture $(p<0.01)$.

The Apoptosis of Human OVCAR-3 and SKOV3 Cells Was Promoted by miR-22 Overexpression To examine whether miR-22 was involved in the apoptosis of human human OVCAR-3 and SKOV3 cells, miR-22 mimics were used to up-regulate the expression level of miR-22 in cells. It was found that the expression of miR-22 was heavily upregulated in both kinds of cells treated with miR-22 mimics after $24 \mathrm{~h}$ of transfection $(p<0.01)$ (Fig. 2A), suggesting that miR-22 mimics were well expressed in cancer cells. In the proliferative study, results showed that cell viability showing as optical density (OD) value of human ovarian cancer cell lines OVCAR-3 and SKOV3 was significantly inhibited by miR-22 overexpression at $48 \mathrm{~h}$ in comparison with the negative control $(p<0.05)$ (Fig. 2B). Furthermore, the apoptotic rates of OVCAR-3 and SKOV3 cells were analyzed using FCM detection kits according to the manufacturer's protocol. Results in Fig. 2C confirmed that the apoptotic rates of OVCAR-3 and SKOV3 were significantly promoted in miR-22 mimic-treated cells in comparison with the negative control $(p<0.01)$.

The Autophagy of Human OVCAR-3 and SKOV3 Cells Was Inhibited by miR-22 Overexpression To further prove the effects of miR-22 on human ovarian cancer cells, the autophagy was analyzed after cells were transfected with the negative control and miR-22 mimics for $48 \mathrm{~h}$. The protein expression of autophagy related proteins Beclin1 and LC3B-II in OVCAR-3 and SKOV3 cells were verified by Western blot. It was suggested that, compared to the control, Beclin 1 and LC3B-II protein expressions were decreased in miR-22 over-

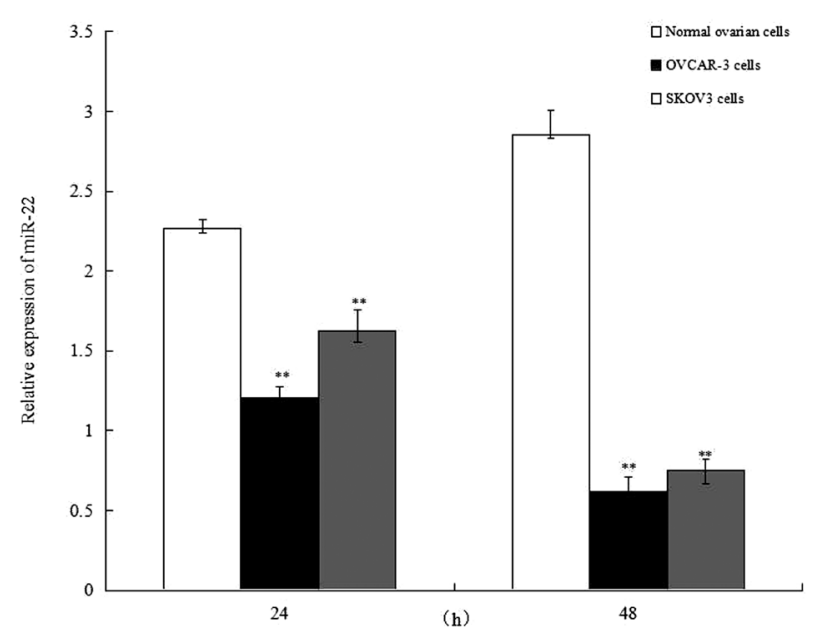

Fig. 1. miR-22 Expression Is Decreased in Human OVCAR-3 and SKOV3 Cells

The expression of miR-22 was measured by RT-PCR in human OVCAR-3 and SKOV3, and normal human ovarian cells after $24 \mathrm{~h}$ and $48 \mathrm{~h}$ of culture. Three individual experiments were performed and each treatment had four replicates. Results were presented as mean \pm S.D. $* * p<0.01$ was considered significant when compared to the control. 

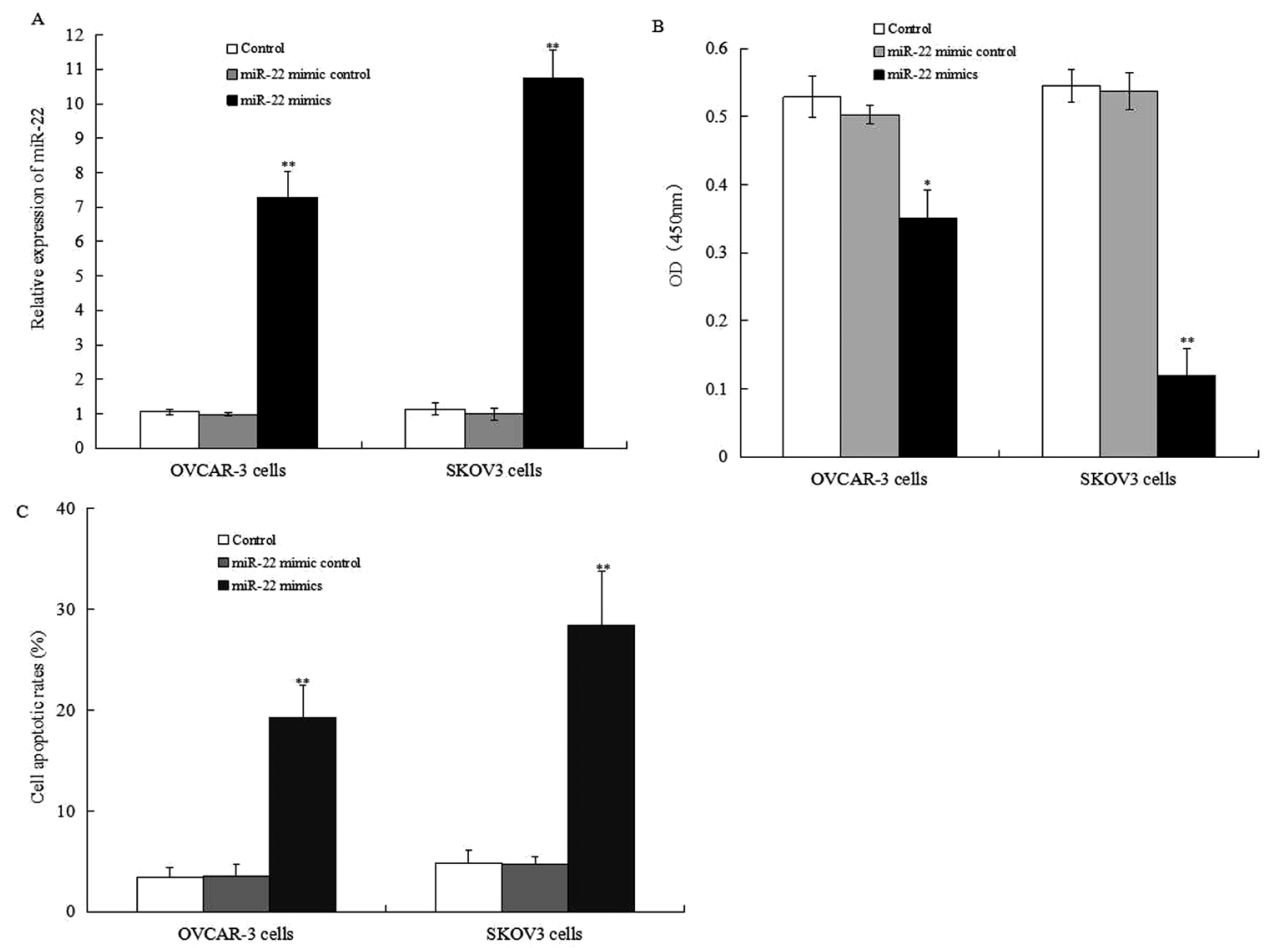

Fig. 2. The Apoptosis of Human OVCAR-3 and SKOV3 Cells Is Promoted by miR-22 Overexpression

After OVCAR-3 and SKOV3 cells were transfected with miR-22 mimics, the expression of miR-22 in human ovarian cancer cells was measured by RT-PCR (A). After cells were treated with the negative mimic control or miR-22 mimics for $48 \mathrm{~h}$, cell viability of human ovarian cancer cells was determined by XTT assay (B), and the apoptotic rate of human ovarian cancer cells was measured by FCM (C). Three individual experiments were performed and each treatment had four replicates. Results were presented as mean \pm S.D. ${ }^{*} p<0.05$ or $* * p<0.01$ was considered significant when compared to the control.
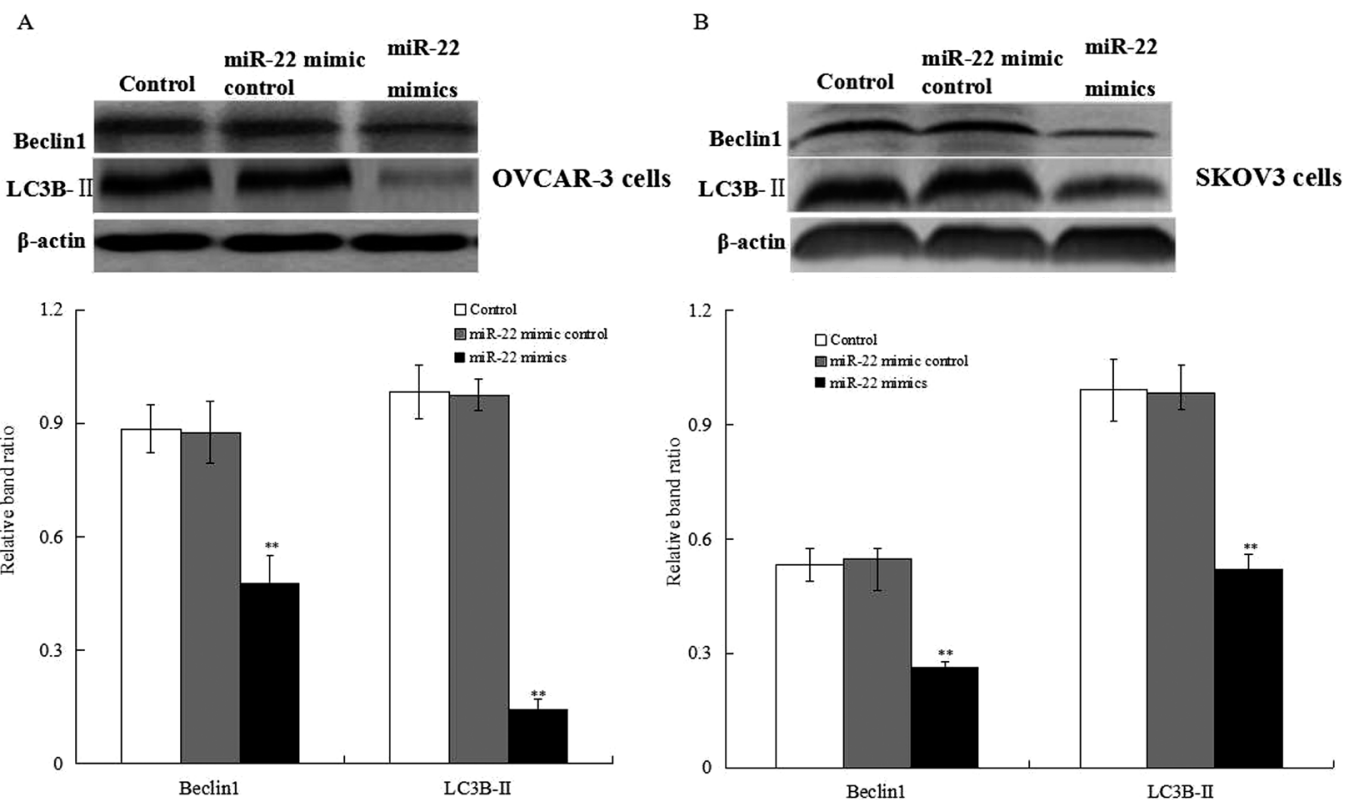

Fig. 3. miR-22 Overexpression Suppresses the Autophagy of Human OVCAR-3 and SKOV3 Cells

Following transfection with the negative mimic control or miR-22 mimic for 48h, and the expression of Beclin1 and LC3B-II in OVCAR-3 (A) and SKOV3 cells (B) was analyzed using Western blot. ${ }^{* *} p<0.01$ was considered significant when compared to the control.

expressing OVCAR-3 cells $(p<0.05)$ (Fig. 3A). Accordingly, Beclin 1 and LC3B-II expression in SKOV3 cells treated with miR-22 mimics was significantly down-regulated $(p<0.05)$ (Fig. 3B).

miR-22 Directly Suppressed the Notch Signal in Human OVCAR-3 and SKOV3 Cells To elucidate the effects of miR-22 on Notch signal, the expression of proteins Notch1 and
Hes1 associated with the Notch signaling pathway was measured using Western blot analysis with and without miR-22 mimic treatment in OVCAR-3 and SKOV3 cells. The results demonstrated that Notch1 and Hes1 protein expression was significantly down-regulated in both OVCAR-3 cells (Fig. 4A) and SKOV3 cells (Fig. 4B) following treatment with miR-22 mimics. It was suggested that over-expression of miR-22 sup- 
A
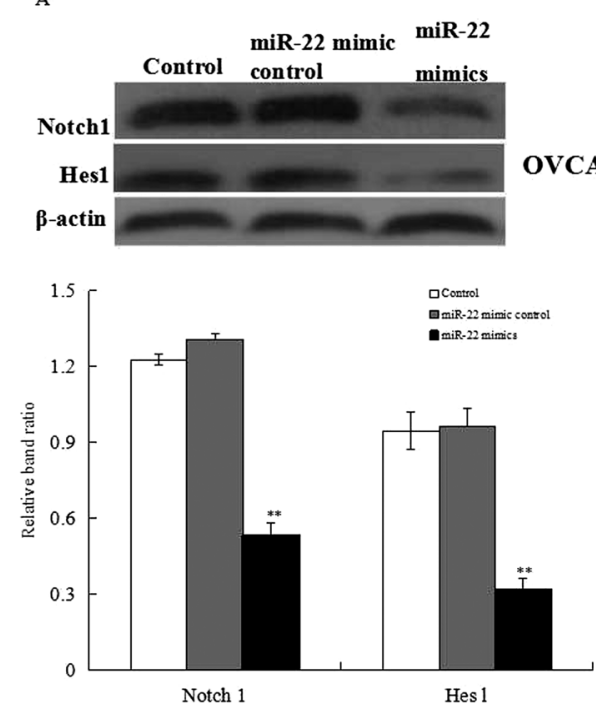
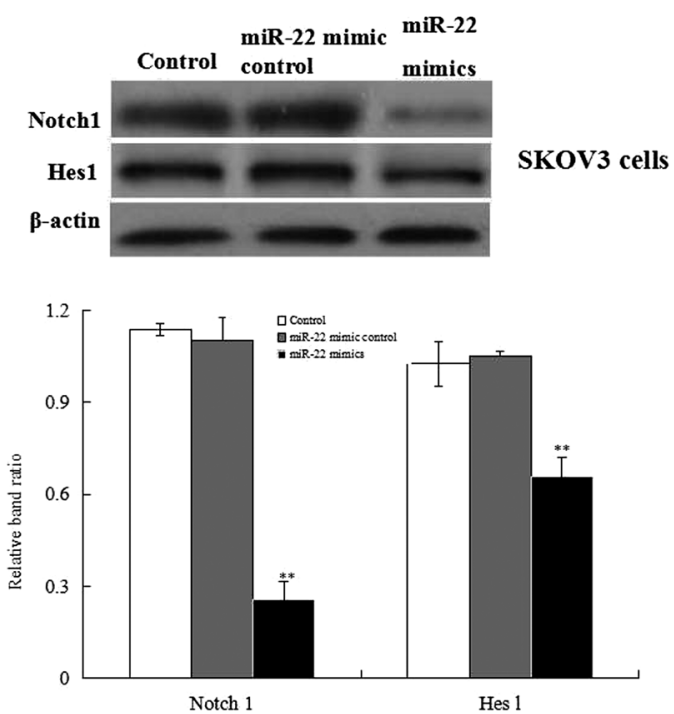

Fig. 4. miR-22 Overexpression Suppresses the Notch Signaling Pathway in Human OVCAR-3 and SKOV3 Cells

After cells were treated with the negative mimic control or miR-22 mimics for $48 \mathrm{~h}$, protein expression of Notch1 and Hes1 was determined by Western blot in OVCAR-3 (A) and SKOV3 cells (B). Three individual experiments were performed and each treatment had three replicates. $* * p<0.01$ was considered significant when compared to the control.
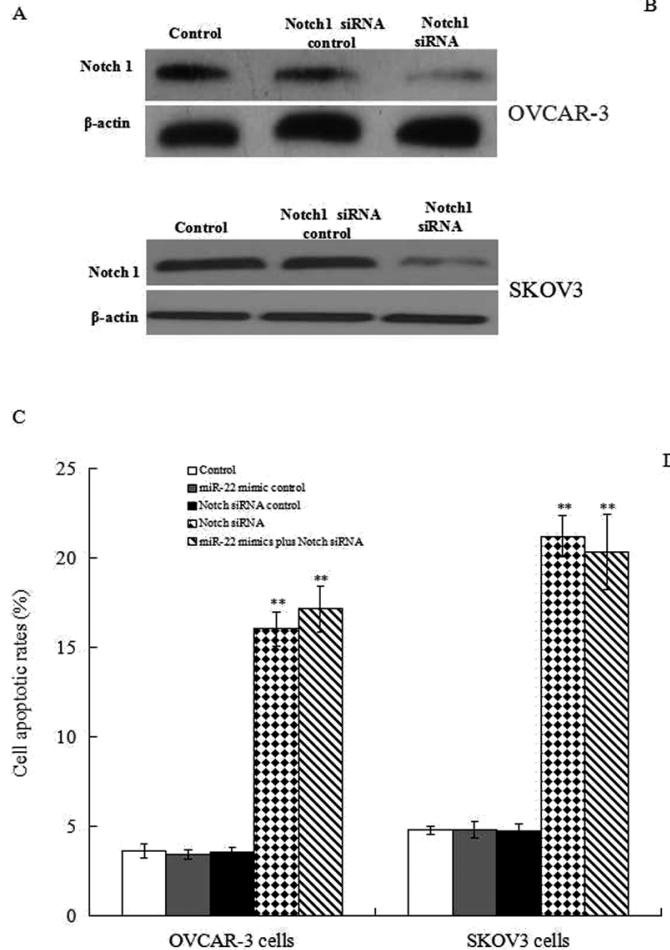

B
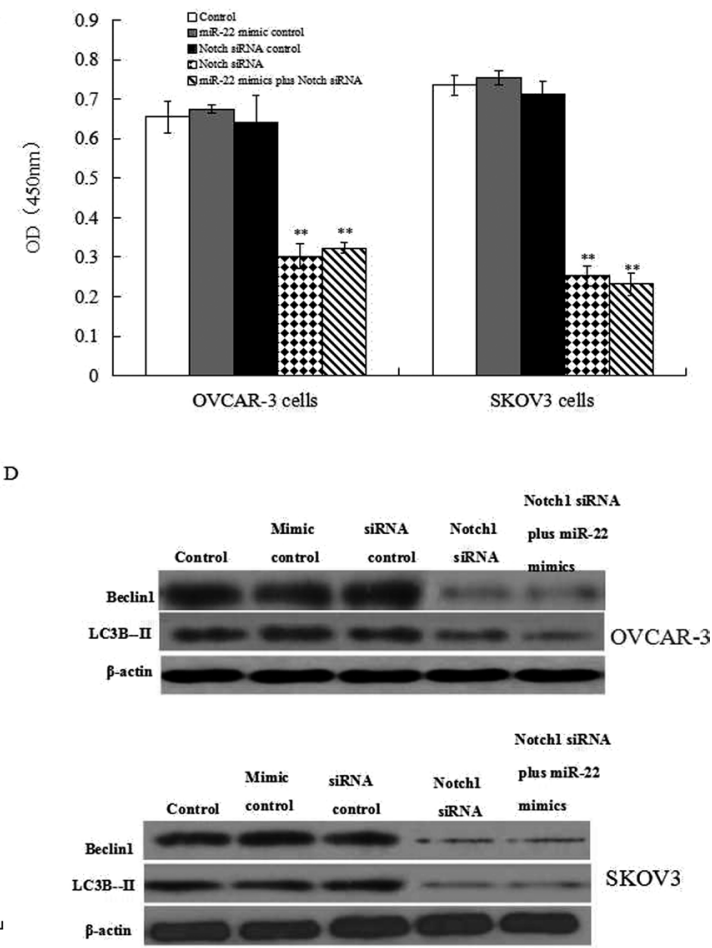

Fig. 5. Effects of miR-22 on Autophagy and Apoptosis in Human OVCAR-3 and SKOV3 Cells Following the Suppression of the Notch Pathway

After blocking the Notch signaling pathway using Notch1 siRNA, protein expression of Notch1 was detected by Western blot (A). After cells were treated with miR-22 mimics, Notch1 siRNA or miR-22 mimics plus Notch1 siRNA, cell viability (B), the apoptotic rate (C) and protein expression of Beclin 1 and LC3B-II (D) were demonstrated. Three individual experiments were performed and each treatment had six replicates. Results were presented as means \pm S.D. ${ }^{*} * p<0.01$ was considered significant when compared to the control.

pressed the activation of the Notch signaling pathway.

The miR-22-Notch Signaling Pathway Was Crucial for the Apoptosis and Autophagy in Human OVCAR-3 and SKOV3 Cells The roles of the Notch signaling pathway in miR-22-induced cellular changes in human ovarian cancer cells were then evaluated by treating with miR-22 mimics and Notch1 siRNA. After OVCAR-3 and SKOV3 cells were treated with Notchl siRNA, the expression level of
Notch1 was heavily inhibited (Fig. 5A). XTT assay and FCM analysis revealed that Notch1 siRNA inhibited cell viability $(p<0.05)$ (Fig. 5B) and activated cell apoptosis $(p<0.05)$ (Fig. 5C) in both OVCAR-3 and SKOV3 cells. However, no synergistic effect was observed in Notch1 siRNA-changed viability and apoptosis following combined treatment with miR-22 $(p>0.05)$. Notch1 siRNA treatment alone suppressed autophagy in OVCAR-3 and SKOV3 cells. Although Notch1 
siRNA inhibited the Notch pathway, no significant differences in Beclin1 and LC3B-II proteins were identified in both OVCAR-3 and SKOV3 cells following combined treatment with miR-22 and Notch1 siRNA when compared with Notch1 siRNA alone $(p>0.05)$ (Fig. 5D).

\section{DISCUSSION}

In various kinds of cancers, miR-22 showed its tumor-suppressive roles, or oncogenic functions. ${ }^{20)}$ In this study, we first attempted to figure out the potential mechanism that miR-22 regulated cellular apoptosis and autophagy in human ovarian lines OVCAR-3 and SKOV3. We finally proved that miR-22 expression was down-regulated in both OVCAR-3 and SKOV3 cells. Furthermore, miR-22 inhibited apoptosis and promoted autophagy of human OVCAR-3 and SKOV3 cells through inhibiting the Notch signaling pathway.

Numerous findings have indicated that miR-22 expression is upregulated or downregulated in different kinds of cancers including prostatic cancer, gastric cancer, breast cancer, esophageal squamous cell carcinoma, osteosarcoma, rhabdomyosarcoma and hepatocellular carcinoma. ${ }^{20)}$ For instance, the lower miR-22 expressions are found in hundreds of clinical samples of the osteosarcoma, rhabdomyosarcoma, prostate, cervical and lung primary tumors. ${ }^{8,12)}$ miR-22 expression detected by RT-qPCR is significantly downexpressed in the osteosarcoma MG-63 cells ${ }^{21)}$ and tissues. ${ }^{11)}$ The expression of miR-22 in hepatocellular carcinoma (HCC) tissues and cell lines are much lower than that in normal control. ${ }^{22}$ ) The expression of miR-22 in esophageal squamous cell carcinoma tissues and cell lines are much lower than that in normal control. ${ }^{23)}$ Compared with normal cerebellum, miR-22 in medulloblastoma tissues and D341, DAOY, ONS-76 medulloblastoma D341, DAOY, ONS-76 cell lines is significantly lower expressed. ${ }^{24)}$ Another study verified the downregulation of miR-22 expression in both glioblastoma tissues and cells but not non-tumor tissues and normal human astrocyte (NHA) cells. ${ }^{25}$ ) In epithelial ovarian cancer, the expression of miR-22 is downregulated. ${ }^{18)}$ Our study also suggests that miR-22 expression is significantly suppressed in both OVCAR-3 and SKOV3 cells compared to normal human ovarian cells. Conversely, in some cases, miR-22 becomes an important activator and its significant upregulation is observed in pancreatic cancer patients, ${ }^{7)}$ and breast tumor samples. ${ }^{26}$

In a review, miR-22 is reported to influence the occurrence and development of some cancers through proliferation, invasion and metastasis through altering its target genes. ${ }^{19)}$ For instance, Gai et $a .^{21)}$ showed that miR-22 overexpression exhibits the obviously decreased proliferation in the MG-63 cells. Additionally, overexpression of miR-22 remarkably increases the apoptosis of the MG-63 cells by elevating Bax expression and reducing Bcl-2 expression. ${ }^{21)}$ Overexpression of miR-22 can significantly inhibit the HCC cell proliferation, migration and invasion in vitro and decrease HCC tumor growth in vivo. ${ }^{22)}$ Transfection of miR-22 mimics can significantly inhibit the cell proliferation, migration and invasion in Eca109 and Kyse410 ESCC cell lines. ${ }^{23)}$ In osteosarcoma cells, miR-22 blocks the high mobility group box 1 (HMGB1)-mediated autophagy, thereby inhibiting the osteosarcoma cell proliferation, migration, and invasion. ${ }^{27)}$ Increasing miR-22 expression inhibits cell proliferation and motility in tongue squamous cell carcinoma TCA8113 cells. ${ }^{28)}$ Transfection of miR-22 mimics can significantly inhibit the increased cell numbers and invasion of A549 and H1299 lung cancer cell lines. ${ }^{29)} \mathrm{We}$ also provides the evidence that miR-22 inhibits cell proliferation and autophagy, and promotes cell apoptosis in human ovarian cancer cell lines OVCAR-3 and SKOV3.

Aberrant signaling pathways are connected with cancer recurrence, metastasis, and treatment resistance. miR-22 has reported to inhibit tumor cell proliferation, migration, and invasion via regulating the signals such as Sirtuin1 (SIRT1) signaling, ${ }^{30,31)}$ YWHAZ/AKT/FOXO3a signaling ${ }^{24)}$ and the cAMP response element binding protein (CREB) and MYC pathways. ${ }^{32}$ Zhen et al. investigated the role and potential mechanism of miR-22 in clear cell ovarian cancer (CCOC) progression and supported that miR-22 is involved in CCOC cell migration and invasion through targeting responsive genes such as CDK6, MDM2, LEF1, MYB, and FOS. ${ }^{13)}$ In the present study, we give a further investigation and demonstrates that the Notch signaling pathway is involved in miR-22-regulated cellular apoptosis and autophagy in human ovarian lines OVCAR-3 and SKOV3. Increasing lines of evidence have suggested that the Notch signaling pathway — an evolutionarily conserved signaling pathway plays a significant role in cellular processes including proliferation, differentiation, and apoptosis. ${ }^{33)}$ Ovarian cancer tissue is also characterized by the high level of Notch1 protein. ${ }^{34)}$ The aberrant expression of Notch pathway is also associated with ovarian carcinoma progression. For example, depletion of Notch1 by Notch1 siRNA significantly inhibited growth of cancer cell lines as $\mathrm{CaOV} 3$, OVCAR3, and SKOV3. ${ }^{35)}$ Our study also indicates that blocking Notch1 expression results in the promotion of cell promotion and autophagy, and the inhibition of apoptosis in human ovarian lines OVCAR-3 and SKOV3, and this effect is also meditated by miR-22.

\section{CONCLUSION}

In summary, we here present that miR-22 is lowly expressed in human ovarian cancer cell lines OVCAR-3 and SKOV3. miR-22 overexpression heavily inhibits cell proliferation and autophagy and enhances cell apoptosis of human ovarian cancer cells by suppressing the Notch signaling pathway. It is indicated that miR-22 functions as tumor suppressors in ovarian cancer, and might become a biomarker and potential therapeutic target in ovarian cancer treatment.

Conflict of Interest The authors declare no conflict of interest.

\section{REFERENCES}

1) Siegel RL, Miller KD, Jemal A. Cancer statistics, 2016. CA Cancer J. Clin., 66, 7-30 (2016).

2) Chandrashekhara SH, Triveni GS, Kumar R. Imaging of peritoneal deposits in ovarian cancer: a pictorial review. World. J. Radiol., 8, 513-517 (2016).

3) Cress RD, Chen YS, Morris CR, Petersen M, Leiserowitz GS. Characteristics of long-term survivors of epithelial ovarian cancer. Obstet. Gynecol., 126, 491-497 (2015).

4) Song SJ, Pandolfi PP. miR-22 in tumorigenesis. Cell Cycle, 13, 11-12 (2014).

5) Li X, Wang S, Chen Y, Liu G, Yang X. miR-22 targets the 3'UTR 
of HMGB1 and inhibits the HMGB1-associated autophagy in osteosarcoma cells during chemotherapy. Tumour Biol., 35, 6021-6028 (2014).

6) Jafarzadeh-Samani Z, Sohrabi S, Shirmohammadi K, Effatpanah H, Yadegarazari R, Saidijam M. Evaluation of miR-22 and miR-20a as diagnostic biomarkers for gastric cancer. Chin. Clin. Oncol., 6, 16 (2017).

7) Hussein NA, Kholy ZA, Anwar MM, Ahmad MA, Ahmad SM. Plasma miR-22-3p, miR-642b-3p and miR-885-5p as diagnostic biomarkers for pancreatic cancer. J. Cancer Res. Clin. Oncol., 143, 83-93 (2017).

8) Bersani F, Lingua MF, Morena D, Foglizzo V, Miretti S, Lanzetti L, Carrà G, Morotti A, Ala U, Provero P, Chiarle R, Singer S, Ladanyi M, Tuschl T, Ponzetto C, Taulli R. Deep sequencing reveals a novel miR-22 regulatory network with therapeutic potential in Rhabdomyosarcoma. Cancer Res., 76, 6095-6106 (2016).

9) Koufaris C, Valbuena GN, Pomyen Y, Tredwell GD, Nevedomskaya E, Lau CH, Yang T, Benito A, Ellis JK, Keun HC. Systematic integration of molecular profiles identifies miR-22 as a regulator of lipid and folate metabolism in breast cancer cells. Oncogene, 35, 2766-2776 (2016).

10) Pasqualini L, Bu H, Puhr M, Narisu N, Rainer J, Schlick B, Schäfer G, Angelova M, Trajanoski Z, Börno ST, Schweiger MR, Fuchsberger C, Klocker H. miR-22 and miR-29a are members of the androgen receptor cistrome modulating LAMC1 and Mcl-1 in prostate cancer. Mol. Endocrinol., 29, 1037-1054 (2015).

11) Wang G, Shen N, Cheng L, Lin J, Li K. Downregulation of miR-22 acts as an unfavorable prognostic biomarker in osteosarcoma. Tumour Biol., 36, 7891-7895 (2015)

12) Xin M, Qiao Z, Li J, Liu J, Song S, Zhao X, Miao P, Tang T, Wang L, Liu W, Yang X, Dai K, Huang G. miR-22 inhibits tumor growth and metastasis by targeting ATP citrate lyase: evidence in osteosarcoma, prostate cancer, cervical cancer and lung cancer. Oncotarget, 7, 44252-44265 (2016)

13) Zhen YB, Guo XL, Xu B, Zhao HW, Xu CJ. Gene expression profiling analysis of the role of miR-22 in clear cell ovarian cancer. Neoplasma, 63, 856-864 (2016).

14) Shawber CJ, Das I, Francisco E, Kitajewski J. Notch signaling in primary endothelial cells. Ann. N. Y. Acad. Sci., 995, 162-170 (2003).

15) Braune EB, Lendahl U. Notch-a goldilocks signaling pathway in disease and cancer therapy. Discov. Med., 21, 189-196 (2016).

16) Cheng P, Kumar V, Liu H, Youn JI, Fishman M, Sherman S, Gabrilovich D. Effects of notch signaling on regulation of myeloid cell differentiation in cancer. Cancer Res., 74, 141-152 (2014).

17) Liu Z, Brunskill E, Varnum-Finney B, Zhang C, Zhang A, Jay PY, Bernstein I, Morimoto M, Kopan R. The intracellular domains of Notch1 and Notch2 are functionally equivalent during development and carcinogenesis. Development, 142, 2452-2463 (2015).

18) Wan WN, Zhang YQ, Wang XM, Liu YJ, Zhang YX, Que YH, Zhao WJ, Li P. Down-regulated miR-22 as predictive biomarkers for prognosis of epithelial ovarian cancer. Diagn. Pathol., 9, 178 (2014).

19) Cheng MJ, Cao YG. TMPYP4 exerted antitumor effects in human cervical cancer cells through activation of p38 mitogen-activated protein kinase. Biol. Res., 50, 24 (2017).

20) Wang J, Li Y, Ding M, Zhang H, Xu X, Tang J. Molecular mechanisms and clinical applications of miR-22 in regulating malignant progression in human cancer. Int. J. Oncol., 50, 345-355 (2017).
21) Gai P, Sun H, Wang G, Xu Q, Qi X, Zhang Z, Jiang L. miR-22 promotes apoptosis of osteosarcoma cells via inducing cell cycle arrest. Oncol. Lett, 13, 2354-2358 (2017).

22) Luo LJ, Zhang LP, Duan CY, Wang B, He NN, Abulimiti P, Lin Y. The inhibition role of miR-22 in hepatocellular carcinoma cell migration and invasion via targeting CD147. Cancer Cell Int., 17, 17 (2017).

23) Yang C, Ning S, Li Z, Qin X, Xu W. miR-22 is down-regulated in esophageal squamous cell carcinoma and inhibits cell migration and invasion. Cancer Cell Int., 14, 138 (2014).

24) Xu QF, Pan YW, Li LC, Zhou Z, Huang QL, Pang JC, Zhu XP, Ren Y, Yang H, Ohgaki H, Lv SQ. MiR-22 is frequently downregulated in medulloblastomas and inhibits cell proliferation via the novel target PAPST1. Brain Pathol., 24, 568-583 (2014).

25) Chen $\mathrm{M}, \mathrm{Hu} \mathrm{W}$, Xiong $\mathrm{CL}, \mathrm{Qu} \mathrm{Z}$, Yin CQ, Wang $\mathrm{YH}$, Luo CL, Guan Q, Yuan CH, Wang FB. miR-22 targets YWHAZ to inhibit metastasis of hepatocellular carcinoma and its down-regulation predicts a poor survival. Oncotarget, 7, 80751-80764 (2016).

26) Damavandi Z, Torkashvand S, Vasei M, Soltani BM, Tavallaei M, Mowla SJ. Aberrant expression of breast development-related MicroRNAs, miR-22, miR-132, and miR-212, in breast tumor tissues. J. Breast Cancer, 19, 148-155 (2016).

27) Guo S, Bai R, Liu W, Zhao A, Zhao Z, Wang Y, Wang Y, Zhao W, Wang W. miR-22 inhibits osteosarcoma cell proliferation and migration by targeting HMGB1 and inhibiting HMGB1-mediated autophagy. Tumour Biol., 35, 7025-7034 (2014).

28) Qiu K, Huang Z, Huang Z, He Z, You S. miR-22 regulates cell invasion, migration and proliferation in vitro through inhibiting CD147 expression in tongue squamous cell carcinoma. Arch. Oral Biol., 66, 92-97 (2016).

29) Ling B, Wang GX, Long G, Qiu JH, Hu ZL. Tumor suppressor miR-22 suppresses lung cancer cell progression through post-transcriptional regulation of ErbB3. J. Cancer Res. Clin. Oncol., 138, 1355-1361 (2012).

30) Chen H, Lu Q, Fei X, Shen L, Jiang D, Dai D. miR-22 inhibits the proliferation, motility, and invasion of human glioblastoma cells by directly targeting SIRT1. Tumour Biol., 37, 6761-6768 (2016).

31) Xiong F, Hu L, Zhang Y, Xiao X, Xiao J. miR-22 inhibits mouse ovarian granulosa cell apoptosis by targeting SIRT1. Biol. Open, 5, 367-371 (2016).

32) Jiang $\mathrm{X}, \mathrm{Hu} \mathrm{C}$, Arnovitz S, Bugno J, Yu M, Zuo Z, Chen P, Huang H, Ulrich B, Gurbuxani S, Weng H, Strong J, Wang Y, Li Y, Salat J, Li S, Elkahloun AG, Yang Y, Neilly MB, Larson RA, Le Beau MM, Herold T, Bohlander SK, Liu PP, Zhang J, Li Z, He C, Jin J, Hong $\mathrm{S}$, Chen J. miR-22 has a potent anti-tumour role with therapeutic potential in acute myeloid leukaemia. Nat. Commun., 7, 11452 (2016).

33) Brzozowa-Zasada M, Piecuch A, Dittfeld A, Mielańczyk Ł, Michalski M, Wyrobiec G, Harabin-Słowińska M, Kurek J, Wojnicz R. Notch signalling pathway as an oncogenic factor involved in cancer development. Contemp. Oncol. (Pozn), 20, 267-272 (2016).

34) Wang M, Wang J, Wang L, Wu L, Xin X. Notch1 expression correlates with tumor differentiation status in ovarian carcinoma. Med. Oncol., 27, 1329-1335 (2010).

35) Rose SL, Kunnimalaiyaan M, Drenzek J, Seiler N. Notch 1 signaling is active in ovarian cancer. Gynecol. Oncol., 117, 130-133 (2010). 